\title{
Enfisema subcutâneo generalizado, decorrente de ferida perfurante em equino
}

\section{Generalized subcutaneous emphysema resulting from perforating wound in equine}

\author{
Yuri da Silva Bonacin ${ }^{1 *}$, Samuel dos Santos Sousa ${ }^{1}$, Gabriela Marchiori Bueno ${ }^{1}$, Amanda Chítero², José Antonio \\ Marques $^{1}$, Paulo Aléscio Canola ${ }^{1}$ \\ ${ }^{1}$ Faculdade de Ciências Agrárias e Veterinárias, Universidade Estadual Paulista (FCAV-Unesp ), Jaboticabal, SP, Brasil \\ ${ }^{2}$ Weems \& Stephens Equine Hospital, Aubrey, Texas, Estados Unidos
}

\section{Resumo}

Enfisema subcutâneo é descrito como o acúmulo de gás de origem multíplice para o espaço subcutâneo, depositando-se entre os feixes e fáscias musculares. Dentre as principais origens, podemos destacar perfurações de traqueia e feridas axilares. Os equinos são altamente suscetíveis a traumas de origem mecânica, tal como lesões por empalamento, choque contra objetos inanimados e brigas entre animais. quando encontradas em locais de movimento contínuo, como axilas, as lesões fazem o movimento de sucção do ar para o espaço subcutâneo, aparecendo nestes locais o sinal clínico clássico, que é a crepitação. Caso não seja instituído tratamento correto, o enfisema subcutâneo progride gradativamente, podendo levar a consequências mais graves como pneumotórax, pnumomediastino e infecções secundárias por bactérias do gênero Clostridium. 0 objetivo deste artigo é relatar o aparecimento de enfisema subcutâneo generalizado em uma égua da raça Quarto de Milha, de 10 anos de idade, causado por ferida axilar no membro torácico esquerdo. Ao exame físico, o animal apresentou todos os parâmetros fisiológicos dentro da normalidade, o que foi observado também no hemograma. 0 tratamento instituído foi à base de limpeza diária da ferida com solução fisiológica e clorexidines degermante e aquoso, com manutenção de uma compressa de gaze introduzida no local, embebida em nitrofurazona com açúcar. Em auxílio à drenagem do gás subcutâneo, foi realizada terapia hídrica e antibioticoterapia sistêmica por três dias, utilizandose penicilina potássica, metronidazol e gentamicina. Houve restrição do movimento, mantendo o animal estabulado durante o tratamento. A alta médica ocorreu após 30 dias. 0 presente relato visa enfatizar a necessidade de um tratamento correto e de rápida escolha, a fim de evitar complicações que possam levar o animal a óbito.

Palavras-chave: Gás. Clostridiose.Crepitação.Tegumento. Tórax. 


\section{Abstract}

Subcutaneous emphysema is described as a gas deposit of multifactorial origin in the subcutaneous space, distributed along the muscle fibers and fascia. The main etiological causes are tracheal rupture and axillary wounds. Horses are highly susceptible to mechanical trauma such as impalement lesions, collision with inanimate objects, and animal fights. These lesions promote suction of gas into the subcutaneous space resulting in a classical clinical sign known as crepitation, especially when they occur around high-motion areas such as the axilla. When the correct treatment is not administered, subcutaneous emphysema can gradually progress, with severe consequences such as pneumothorax, pneumomediastinum, and secondary infection caused by Clostridium species. The aim of this paper is to report generalized subcutaneous emphysema in a ten-year-old American Quarter Horse mare caused by a wound located in the axilla of the left forelimb. At physical examination, the animal presented normal physical parameters and hemogram values. The treatment included daily wound care with sterile solution, chlorhexidine digluconate, and aqueous chlorhexidine. A gauze imbibed in nitrofurazone and containing sugar blend was inserted in the wound. Daily hydrotherapy was combined with antibiotics (penicillin potassium, metronidazole, and gentamicin) to help with the gas drainage. Further, exercise restriction was instituted with the animal kept in the stall for 30 days. This case report lays emphasis on correct and prompt treatment with a focus on preventing complications that could lead to a fatal outcome.

Keywords: Gas. Clostridiosis. Crepitation. Tegument.Thorax.

\section{Introdução}

Descrito como o acúmulo de gás no espaço subcutâneo, resultante principalmente de lesões traqueais e lacerações axilares (Hance e Robertson, 1992), o enfisema subcutâneo em equinos é raro. As feridas axilares são geralmente originárias de diversas lesões traumáticas como, por exemplo, choque dos animais contra objetos inanimados, empalamento por objetos pontiagudos como cercas, galhos de árvore e partes de metal dos trailers de transporte, disputas entre animais, os quais podem desferir coices, e até mesmo ferimentos causados por animais com cornos (Laverty et al., 1996; Sprayberry e Barrett, 2015). As feridas no momento inicial podem se revelar como pequenos traumas, porém necessitam de monitoramento contínuo a fim de evitar o desenvolvimento de complicações graves (Joswig e Hardy, 2013).

Em humanos, o enfisema subcutâneo possui três etiologias: (1) infecção local com proliferação de microrganismos formadores de gás; (2) oriundo de lesões traumáticas locais por quebra da homeostase cutânea; (3) enfisema espontâneo, onde o aumento da pressão intra-alveolar é ultrapassado, causando ruptura alveolar e estravazamento do ar para o espaço intersticial e subcutâneo (Maunder et al., 1984). Estas etiologias ocorrem de maneira semelhante nos equinos, com adendo anatômico do mediastino com pequenas fenestrações, o que pode causar pneumotorax bilateral nesta espécie (Boy e Sweeney, 2000).

Nas feridas axilares, o que mais ocorre é a forma (2), onde o ar ambiente é bombeado através da ferida, quando o animal se movimenta, por um mecanismo semelhante ao de uma válvula de uma via. Quando o acúmulo de gás se faz de forma generalizada, a musculatura torácica tem extrema força contrátil, porém possui pequenas fissuras nas fáscias musculares, tornando possível a passagem de gás para o mediastino (Hance e Robertson, 1992; Joswig, 2013). Com a ocorrência de pneumomediastino, os animais podem desenvolver pneumotórax (Joswig, 2013). Outras consequências comuns das feridas axilares são a incorporação de corpo estranho, fístulas, pleurite e pleuropneumonia (Saprayberry e Barret, 2015). Segundo Hance e Robertson (1992), a complicação menos severa do enfisema subcutâneo é a desfiguração cosmética temporária, porém pode ocorrer obstrução das vias áereas superiores pelo acúmulo de gás em espaço retrofaringeo, cegueira por compressão do segundo par de nervos cranianos (nervo óptico) e déficit respiratório.

As contaminações secundárias por bactérias que produzem gás, como as do gênero Clostridium, podem ser a causa do enfisema subcutâneo, devido ao ambiente anaeróbio propício, gerando mionecrose aguda (Marble et al., 1996; Peek et al., 2003).

0 tratamento do enfisema subcutâneo pode demorar semanas e é baseado no desbridamento da 
ferida, lavagem com antissépticos e fechamento por primeira intenção ou colocação de gaze no interior da ferida, bandagem e cicatrização por segunda intenção. A antibioticoterapia sistêmica de amplo espectro também deve ser instituída (Laverty et al., 1996; Sprayberry e Barret, 2015). Para auxiliar o tratamento, podem ser realizadas pequenas incisões de pele a fim de promover drenagem do gás rapidamente. A restrição de movimento do animal deve ser instituída até a oclusão total da ferida, a fim de interromper a sucção de ar para o espaço subcutâneo (Joswig e Hardy, 2013).

Com o aumento da tecnificação e manejo intensivo dos equinos, os animais convivem progressivamente com instalações e outros objetos inanimados que, devido ao temperamento sanguíneo desta espécie animal, potencialmente geram o risco de choques mecânicos e lacerações da pele. Apesar de o aparecimento do enfisema subcutâneo ser incomum, a instituição de um tratamento rápido, correto e eficaz pode impedir que os animais acometidos sofram de complicações graves, as quais podem levar a óbito. 0 objetivo do presente relato é apresentar um caso clínico e o sucesso no tratamento, o qual pode ser seguido em outros casos semelhantes, atentando para as possíveis complicações.

\section{Relato do caso}

Um equino da raça Quarto de Milha, de 10 anos de idade, fêmea, foi referida ao Hospital Veterinário Governador Laudo Natel, da Unesp de Jaboticabal, com queixa de aumento de volume generalizado em espaço subcutâneo e ferida na região axilar do membro torácico esquerdo.

Segundo relato do proprietário, após o trabalho, foi observada uma ferida perfurante na região axilar esquerda, na altura da articulação húmero-rádioulnar, medial à tuberosidade do olecrano, entre a porção média do músculo peitoral ascendente e a inserção da cabeça longa do músculo tríceps braquial. Houve aumento de volume regional e presença de sangue. Foi realizada, na propriedade, limpeza da ferida com peróxido de hidrogênio e iodo povidona tópico durante duas semanas prévias ao encaminhamento.
Ao exame físico, o animal apresentou todos os parâmetros fisiológicos dentro da normalidade. Foi realizado hemograma, o qual não demonstrou nenhuma alteração.

$\mathrm{Na}$ inspeção visual, verificou-se que o animal possuía aumento de volume subcutâneo generalizado, estendendo-se da face até a vulva, conforme Figura 1. Na palpação manual, confirmouse a suspeita clínica de enfisema subcutâneo. A ferida possuía $5 \mathrm{~cm}$ de comprimento por $3 \mathrm{~cm}$ de diâmetro, e apresentava drenagem de conteúdo serosanguinolento de odor fétido (Figura 2).

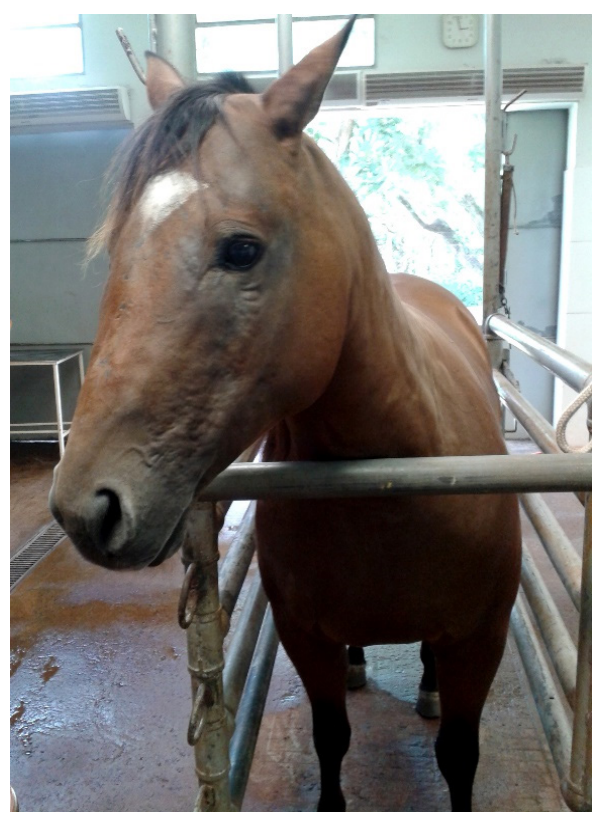

Figura 1 - Equino apresentando enfisema subcutâneo generalizado e deformação cosmética da face, decorrente de ferida axilar.

0 animal apresentava hiperalgesia à pressão digital e, portanto, foi realizada sedação com cloridrato de xilazina $10 \%$ na dose de $0,5 \mathrm{mg} / \mathrm{kg}$ pela via endovenosa, para a exploração da ferida. Em seguida, foi realizada a limpeza com clorexidines degermante $4 \%$ e aquoso $2 \%$, e solução fisiológica de $\mathrm{NaCl}$ 0,9\% a fim de remover a secreção. Na inspeção não foi encontrado corpo estranho e o tratamento instituído foi baseado na antibioticoterapia, drenagem do enfisema e fechamento da ferida por segunda intenção. 


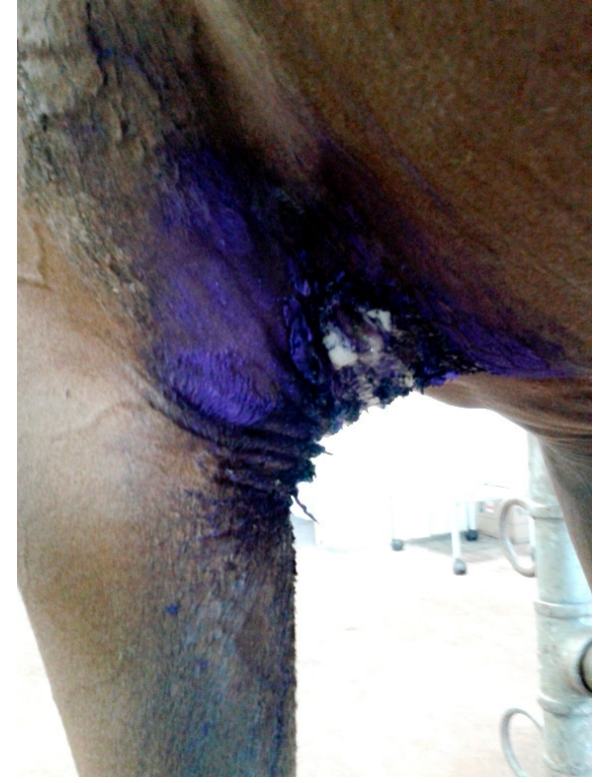

Figura 2 - Vista Lateral da região axilar esquerda de equino, apresentando ferida e drenagem de conteúdo serosanguinolento.

Foram realizadas limpezas diárias do exterior da ferida com solução estéril de $\mathrm{NaCl}$ 0,9\%, clorexidine aquoso $2 \%$ e gaze estéril, com posterior introdução de uma compressa de gaze tipo queijo, embebida em solução de nitrofurazona com açúcar. Foi aplicada ducha de água fria uma vez ao dia, realizando a drenagem do enfisema no sentido das extremidades do animal para o local da ferida. Foi realizada antibioticoterapia sistêmica por três dias, com uso de penicilina potássica na dose de 30.000 $\mathrm{UI} / \mathrm{kg}$, a cada 6 horas, associada ao metronidazol na dose de $15 \mathrm{mg} / \mathrm{kg}$ a cada 12 horas, e sulfato de gentamicina na dose de $6,6 \mathrm{mg} / \mathrm{kg}$ a cada 24 horas, todos por via endovenosa. Durante o tratamento, o animal foi mantido na baia de internação e teve alta médica em 30 dias.

\section{Discussão}

Como já descrito amplamente na literatura, as principais causas de feridas axilares em equinos são o choque mecânico contra objetos inanimados ou mesmo lesões traqueais (Laverty et al., 1996; Sprayberry e Barrett, 2015). Desta forma, todos os autores corroboram com o histórico apresentado pelo proprietário do animal, onde a ferida axilar foi traumática, funcionando como válvula de sucção única do ar para todo o espaço subcutâneo. Alguns animais desenvolvem o enfisema gradativo, com início do quadro em três dias (Joswig e Hardy, 2013), o qual pode evoluir para um quadro generalizado em sete a 16 dias (Laverty et al., 1996; Joswig e Hardy, 2013). No animal atendido houve aumento de volume local inicial, porém acredita-se que foi decorrente de reação inflamatória, e o enfisema ocorreu gradativamente em 14 dias, período descrito na literatura.

Além do exame clínico detalhado, a exploração da ferida é uma ferramenta diagnóstica importante, visto que a presença de exsudato de odor fétido pode indicar infecção secundária por bactérias produtoras de gás, como as do gênero Clostridium (Marble et al., 1996). A ferida axilar apresentava drenagem de conteúdo fétido, no entanto não houve nenhuma alteração hematológica ou clínica sugestiva de clostridiose. Em casos de mionecrose bacteriana, a dosagem sérica de creatina fosfoquinase (CK) é uma importante ferramenta de diagnóstico e acompanhamento, conforme descrevem Martins e colaboradores (2018), porém foi um fator limitante durante o tratamento.

Devido à grande disseminação do gás pelo espaço subcutâneo, alguns autores recomendam a realização de fenestras por meio de bisturi, auxiliando na drenagem de gás (Joswig e Hardy, 2013), técnica evitada pelos autores deste relato pois houve boa evolução do caso com tratamento conservativo. Optou-se pela terapia hídrica, com duchas diárias, na tentativa de auxiliar a drenagem do gás para a ferida, técnica também descrita por Laskoski e colaboradores (2015), obtendo-se resultado satisfatório. Vale ressaltar que o animal apresentava leve desconforto durante a realização deste procedimento, informação a qual não foi relatada por outros autores.

O tratamento baseado na limpeza local com antisséptico e colocação de gaze estéril é imperativo em todos os relatos encontrados na literatura, com alguns autores recomendando a aplicação de bandagens na região axilar (Joswig e Hardy, 2013) ou mesmo suturas de ancoragem (Laskoski et al., 2015). No presente relato, devido às dimensões da ferida e extensa profundidade, apenas a inserção de 
gaze tipo queijo foi suficiente para iniciar o processo de reparação tecidual.

Como as feridas axilares fazem sucção do ar para o subcutâneo, um excelente complemento ao tratamento é a restrição do movimento, mantendo o animal estabulado (Joswig, 2013), tal como se procedeu com o equino durante o tratamento.

A terapia antimicrobiana de escolha foi a penicilina potássica associada à gentamicina e metronidazol, porém existem relatos do uso de sulfa trimetropim, penicilina procaína, gentamicina e cloranfenicol (Joswig e Hardy, 2013) ou apenas metronidazol (Laverty et al., 1996).

Em traumas extensos onde pode ocorrer pneumotorax, penumomediastino ou outras alterações pulmonares, com mudanças na coloração de mucosas, o exame clínico deve ser detalhado a fim de identificar possíveis complicações (Boy e Sweeney, 2000). O equino acometido não apresentou nenhuma alteração sistêmica, apenas abafamento de sons pulmonares pela crepitação local, corroborando com os resultados obtidos por Boy e Sweeney (2000).

O prognóstico para casos de enfisema subcutâneo é bom. Em casos menos graves, a absorção e resolução clínica podem ser espontâneas (Marble et al., 1996). 0 animal do presente relato recebeu alta médica com 30 dias de tratamento, período descrito por alguns autores (Hance e Robertson, 1992; Laverty et al., 1996).

\section{Conclusão}

Os casos de enfisema subcutâneo generalizado são raros, porém o retardo na implementação do tratamento ideal pode acarretar em complicações secundárias graves, tal como pneumotórax. No presente relato, o tratamento foi implantado com sucesso, baseado na limpeza da ferida, terapia antimicrobiana e confinamento do animal, podendo ser utilizado para casos semelhantes. Recomendase o acompanhamento intensivo a fim de detectar precocemente qualquer alteração clínica que possa colocar a vida do animal em risco.

\section{Referências}

Boy MG, Sweeney CR. Pneumothorax in horses: 40 cases (1980-1997). J Am Vet Med Assoc. 2000;216(12):1955-9.

Hance SR, Robertson JT. Subcutaneous emphysema from axillary wound that resulted in pneumomediastinum and bilateral pneumothorax in a horse. J Am Vet Med Assoc. 1992;200(8):1107-10.

Joswig A, Hardy, J. Axillary wounds in horses and the development of subcutaneous emphysema, pneumomediastinum and pneumothorax. Equine Vet Educ. 2013;25(3):139-43.

Joswig AJ. How to manage axillary wounds. AAEP Proceedings. 2013;59:485-8.

Laskoski LM, Fioravanti RAB, Doria RGS, Carvalho MB, Freitas SH. Enfisema subcutâneo generalizado em um equino após ferida axilar - Relato de caso. Rev Bras Med Vet. 2015;37(1):93-6.

Laverty S, Lavoie JP, Pascoe JR, Ducharme N. Penetrating wounds of the thorax in 15 horses. Equine Vet J. 1996;28(3):220-4.

Marble SL, Edens LM, Shiroma JT, Savage CJ. Subcutaneous emphysema in a neonatal foal. J Am Vet Med Assoc. 1996;208(1):97-9.

Martins MC, Bathe AP, Marr CM. Pneumopericardium associated with blunt thoracic trauma in an adult horse. Equine Vet Educ. 2018;30(7):346-51.

Maunder RJ, Pierson DJ, Hudson LD. Subcutaneous and mediastinal emphysema. Pathophysiology, diagnosis, and management. Arch Intern Med. 1984;144(7):1447-53.

Peek SF, Semrad SD, Perkins GA. Clostridial myonecrosis in horses (37 cases 1985-2000). Equine Vet J. 2003;35(1):86-92.

Sprayberry KA, Barrett EJ. Thoracic trauma in horses. Vet Clin North Am Equine Pract. 2015;31(1):199-219. 